\title{
Für fast alle Inkretine laufen heute kardiovaskuläre Endpunktstudien
}

\begin{abstract}
In den nächsten sechs Jahren werden die Resultate von sieben Endpunktstudien mit über 66000 Patienten erwartet, in denen die kardiovaskulären Effekte von DPP4-Hemmern und Inkretinmimetika untersucht werden.
\end{abstract}

\begin{tabular}{|c|c|c|c|c|}
\hline Klasse & Medikament & Studie & Patienten & Studienende \\
\hline DPP-4 & Hemmer & Savor-TIMI 53 & 16500 & 2014 \\
\hline \multirow[t]{3}{*}{ Hemmer } & Alogliptin & Examine & 5400 & 2015 \\
\hline & Linagliptin & Carolina & 6000 & 2018 \\
\hline & Sitagliptin & Tecos & 14000 & 2014 \\
\hline GLP-1- & Exenatide & Exscel & 9500 & 2017 \\
\hline Rezeptor- & Liraglutide & Leader & 8754 & 2016 \\
\hline Agonisten & Lixisenatide & Elixa & 6000 & 2014 \\
\hline
\end{tabular}

Antidiabetika sind früher auf Basis ihrer Blutzuckersenkung zugelassen worden, berichtet die Diabetologin Prof. PetraMaria Schumm-Draeger vom Klinikum München-Bogenhausen. Heute verlangen wichtige Institutionen wie die US-Zulassungsbehörde FDA, dass für neue Antidiabetika kardiovaskuläre Langzeitdaten erhoben werden.

Dies ist notwendig geworden, weil einige Antidiabetika - Beispiele sind Rosiglitazon oder Sulfonylharnstoffe - mit einem erhöhten kardiovaskulären Risiko in Verbindung gebracht wurden. Bei sehr scharfer Blutzuckereinstellung hatten sich zudem in einigen Studien ungünstige kardiovaskuläre Effekte gezeigt.

Positives Resultat der strikteren Behördenpolitik ist, dass beinahe alle Inkretinbasierten Medikamente in kardiovaskulären Langzeitstudien getestet werden (Tabelle 1).

Weit fortgeschritten ist bereits die SAVOR-TIMI-53-Studie mit 16500 Patienten, die entweder kardiovaskuläre Erkrankungen oder multiple kardiovaskuläre Risikofaktoren aufweisen. Studienmedikament ist Saxagliptin (Onglyza ${ }^{\circledR}$ ). Primärer Endpunkt sind kardiovaskulärer Tod, Herzinfarkt oder Schlaganfall. Untersucht wird auch die Langzeitsicherheit, sprich Hypoglykämien, Krebsrate, Nebenwirkungen, sowie der Effekt bei Patienten mit Nierenschwäche in halber
Dosis. Die Ergebnisse werden 2014 erwartet.

Bisher waren unter Saxagliptin in einer Metaanalyse von acht Phase-2-3-Studien mit 4607 Patienten deutlich weniger kardiovaskuläre Ereignisse aufgetreten als unter Placebo, so Schumm-Draeger. Allerdings war die Behandlungsdauer jeweils kurz und die Ereignisrate insgesamt gering.

DPP-4-Inhibitoren wie Saxagliptin reduzieren das $\mathrm{HbA}_{1 \mathrm{c}}$ um etwas mehr als $0,5 \%$, indem sie nach den Mahlzeiten die Sekretion von Insulin fördern und diejenige von Glukagon reduzieren. Zu ihren Vorteilen gehört, dass sie oral eingenommen und gastrointestinal gut vertragen werden, keine Gewichtszunahme und selten Hypoglykämien verursachen, sagte der Diabetologe Prof. Jiten Vora vom Royal Liverpool University Hospital. Bei Niereninsuffizienz sind sie in reduzierter Dosis anwendbar. Saxagliptin wird ab einer Kreatinin-Clearance unter $50 \mathrm{ml} /$ min in der Dosis von $5 \mathrm{mg} / \mathrm{d}$ auf 2,5 mg/d halbiert.

Dr. med. Dirk Einecke, München

Quelle: Satelliten-Symposium "Delivering Innovation in Type 2 Diabetes: Tailored approaches with SGLT2 and incretin-based therapies" bei der Jahrestagung der European Association for the Study of Diabetes (EASD) in Berlin, 1. Oktober 2012, Veranstalter: BMS und Astra Zeneca

\section{Hämoglobinspray bringt Sauerstoff in chronische Wunden}

Hypoxie ist bei der Entstehung vieler chronischer Wunden beteiligt und kann die Heilung behindern. Moderne Therapieansätze zielen daher darauf $\mathrm{ab}$, die Sauerstoffversorgung in den Wunden zu verbessern.

$\mathrm{Zu}$ den häufigsten chronischen Wunden zählen das Ulcus cruris venosum, Fußverletzungen bei peripherer arterieller Verschlusskrankheit und Diabetes mellitus sowie der Dekubitus. Bei allen kommt es zur Hypoxie, die die verschiedenen Phasen der Wundheilung negativ beeinflussen kann.

Hypoxie verringert in der Reinigungs- und Entzündungsphase der Wundheilung das Zellwachstum und die Proteinsynthese. In der Proliferations- und Epithelisierungsphase stört die Hypoxie etwa die Angiogenese, die Synthese und Vernetzung von Kollagen sowie letztlich den Wundverschluss. Auch die abschließende Gewebeumbauphase wird wesentlich durch das Sauerstoffangebot beeinflusst und läuft bei einem Mangel eventuell nicht korrekt ab.

Für die Behandlung chronischer Wunden kann eine ausreichende Sauerstoffversorgung demnach wichtig sein. Zur Verbesserung des lokalen Sauerstoffangebots chronischer Wunden gibt es verschiedene Behandlungsansätze. Dazu gehört die hyperbare Sauerstofftherapie, für die allerdings eine begehbare Druckkammer nötig ist. Daneben gibt es Geräte zur topischen Behandlung. Zudem sind Zubereitungen verfügbar, $\mathrm{zu}$ denen sauerstoffgenerierende Wundauflagen oder das Hämoglobinspray Granulox gehören. Das Spray nutzt wasserlösliches Hämoglobin, das sich gleichmäßig im Wundexsudat verteilt. Das Hämoglobin bindet nach dem Aufsprühen Luftsauerstoff und transportiert ihn entlang des Konzentrationsgefälles zum Wundgrund.

Bislang konnten in Studien mit diesen Therapiemethoden viel versprechende Ergebnisse erzielt werden.

Quellen: Anne-Kristin Schulze nach Kröger K et al. Wund Management 2012/05: 212-7 und www.granulox.de 